\title{
Modelling the Influence of Awareness Programs by Media on the Drinking Dynamics
}

\author{
Hai-Feng Huo and Qian Wang \\ Department of Applied Mathematics, Lanzhou University of Technology, Lanzhou, Gansu 730050, China \\ Correspondence should be addressed to Hai-Feng Huo; huohf1970@gmail.com
}

Received 25 January 2014; Accepted 17 March 2014; Published 16 April 2014

Academic Editor: Yongli Song

Copyright ( 2014 H.-F. Huo and Q. Wang. This is an open access article distributed under the Creative Commons Attribution License, which permits unrestricted use, distribution, and reproduction in any medium, provided the original work is properly cited.

\begin{abstract}
We develop a nonlinear mathematical model with the effect of awareness programs on the binge drinking. Due to the fact that awareness programs are capable of inducing behavioral changes in nondrinkers, we introduce a separate class by avoiding contacts with the heavy drinkers. Furthermore we assume that cumulative density of awareness programs increases at a rate proportional to the number of heavy drinkers. We establish some sufficient conditions for the stability of the alcohol free and the alcohol present equilibria and give some numerical simulations to explain our main result. Our results show that awareness programs is an effective measure in reducing alcohol problems.
\end{abstract}

\section{Introduction}

Alcohol consumption, particularly heavier drinking, is an important risk factor for many health problems and is a major contributor to the global burden of disease [1]. It has been identified as an important risk factor for chronic disease and injury [2]. Drinking alcohol causes the liver to become even more damaged in people infected with the hepatitis B virus (HBV) [3]. The CAS (Central Authentication Service) findings have shown that alcohol consumption at binge levels and beyond has a significant impact on college students' academic performance, social relationships, risk taking behaviors, and health. This form of drinking is associated with missing class, falling behind in schoolwork, and lower grade point average, a relationship mediated by fewer hours spent in studying [4, 5]. In addition, students who attended schools with high rates of binge drinking experienced a greater number of secondhand effects, including disruption of sleep or study, property damage, and verbal, physical, or sexual violence, than their peers attending schools with low binge drinking rates [6]. Prior studies have indicated that heavy alcohol drinkers are likely to engage in risky sexual behaviours and, thus, more likely to get sexually transmitted infections (STIs) than social drinkers [7]. The rapid consumption of large amounts of alcohol, especially by young people, leads to serious antisocial and criminal behavior in urban centres. This phenomenon has grown very rapidly [8]. Since mathematical model is a predictive tool which can mimic the process of drinking and provide useful tools to analyze the spread and control of drinking behavior, several different mathematical models for drinking have been formulated and studied recently. Simple models for alcohol treatment are presented by Sanchez et al. [9] which were based on studying binge drinking in a college system and assumed the same "leaving rate." Mulone and Straughan [10] investigated a possible model for binge drinking taking into account admitting and nonadmitting drinkers. But the global analysis of binge drinking model is not discussed in the literature. Huo and Song [11] introduced a more realistic two-stage model for binge drinking problem, where the youths with alcohol problems are divided into those who admit the problem and those who do not admit it. For the other mathematical models for drinking or smoking, we refer to [12-14] and the references cited therein.

The media may be the most important source of health information for the general public; it can play a special role in providing a voice for people to express their experiences of illness [15]. Jaramillo [16] studied a model presenting an evaluation of the impact on case finding of a mass media 
health education campaign for TB control in Cali, Colombia. Liu et al. [17] incorporate the psychological impacts of media coverage on disease transmission and emphasize the importance of refining classical mathematical models to reflect the partially self-limiting nature of infectious disease outbreaks, due to the effects of widespread news coverage and fast information flow. Web-based screening and brief interventions that include personalized feedback about their alcohol use have proven to be particularly promising for reducing hazardous drinking among university students [18]. Mass media (television, radio, newspapers, billboards, and booklets) have been used as a way of delivering preventive health messages as they have the potential to influence people's behavior. Further, mass media have been deployed in the effort to control and eliminate epidemic diseases [19]. Xiang et al. [20] studied a drinking model with public health educational campaigns which can be an efficient option for reducing the spread of disease. Misra et al. [21, 22] considered the effects of awareness programs driven by media on the spread of infectious diseases; their results showed that, though awareness programs cannot eradicate infection, they help in controlling the prevalence of disease. The most successful approaches concentrate not on trying to change the drinker's behavior directly but on influencing the community environment around the nondrinker. For instance, education and persuasion may increase knowledge and change attitudes but have no effect on drinking [23,24], so we only consider the interaction from awareness programs between aware individual and nondrinker.

Motivated by the above works, we introduce a mathematical model with the effect of awareness programs on the binge drinking in this paper. Because awareness programs are capable of inducing behavioral changes in susceptible individuals, we introduce a separate class $X(t)$ of those who are aware of risk and refrain from drinking by avoiding contact with the heavy drinkers. This newly formed aware class is assumed to be fully protected from the heavy drinkers due to media-induced isolation and individuals of this class may contact heavy drinkers only if they lose awareness $[21,22]$. Furthermore, we assume that cumulative density of awareness programs $M(t)$ increases at a rate proportional to the number of heavy drinkers. We establish some sufficient conditions for the global stability of equilibria and give some numerical simulations to explain our main result. Our results show that awareness programs are an effective measure in reducing alcohol problems.

The organization of this paper is as follows. In the next section, the effect of awareness programs by media on binge drinking model is formulated. In Section 3, the existence and the stability of equilibria are investigated. Some numerical simulations are given in Section 4. Some discussions are given in Section 5.

\section{The Model}

2.1. System Description. The total population is divided into five compartments, namely, the susceptible compartment who do not drink or drink only moderately, denoted by $S(t)$, those who are aware of risk and avoid drinking, denoted by

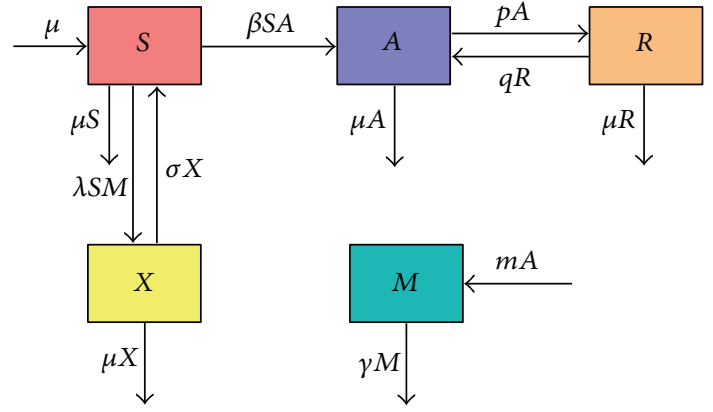

Figure 1: Transfer diagram of model (2).

$X(t)$, those who drink heavily, denoted by $A(t)$, and those who are in treatment, denoted by $R(t)$. Also let $M(t)$ be the cumulative density of awareness programs driven by media. The growth rate of density of awareness programs is assumed to be proportional to the number of heavy drinkers. It is considered that, due to the awareness programs, nondrinkers form a different class and avoid contacting with the heavy drinkers. And we only consider the interaction from awareness programs between aware individual and nondrinker. The total number of population at time $t$ is given by

$$
N(t)=S(t)+X(t)+A(t)+R(t) .
$$

The model structure is shown in Figure 1. The transfer diagram leads to the following system of ordinary differential equations:

$$
\begin{aligned}
& \frac{d S(t)}{d t}=\mu-\beta S A-\lambda S M+\sigma X-\mu S, \\
& \frac{d X(t)}{d t}=\lambda S M-\sigma X-\mu X, \\
& \frac{d A(t)}{d t}=\beta S A+q R-p A-\mu A, \\
& \frac{d R(t)}{d t}=p A-q R-\mu R, \\
& \frac{d M(t)}{d t}=m A-\gamma M,
\end{aligned}
$$

where $\mu$ is the rate of individuals entering into the system in a given time interval (say, each year). Since we are dealing with youths, we assume that the death rate is negligible and so the leaving rate is also $\mu$. This is in accordance with an assumption by Sanchez et al. [9], where "leaving rate" presents the departure rate from drinking environment. $\beta$ is the transmission coefficient for the nondrinkers turning to heavy drinkers through peer pressure. $\lambda$ represents the dissemination rate of awareness among nondrinkersdue to the awareness programs, nondrinkers form a different class $X(t)$ and avoid contacting with the heavy drinkers. The constant $\sigma$ denotes the rate of transfer of aware individuals to nondrinker class. $p$ is a proportionality coefficient for $A$ going into treatment. $q$ is a proportionality coefficient for 
$R$ relapsing into drinkers. $m$ represents the growth rate of density of awareness programs which is assumed to be proportional to the number of heavy drinkers, and $\gamma$ represents the depletion rate of these programs due to ineffectiveness, social problems, and so forth. For instance, as time passes by, some of the campaigns by media lose their influence or fail to attract people.

\subsection{Basic Properties}

\subsubsection{Invariant Region}

Lemma 1. All feasible regions $\Omega$ defined by

$$
\begin{aligned}
\Omega=\{ & (S(t), X(t), A(t), R(t), M(t)) \in R_{+}^{5}: \\
& \left.S(t)+X(t)+A(t)+R(t) \leq 1 ; M(t) \leq \frac{m}{\gamma}\right\}
\end{aligned}
$$

with the initial conditions $S(0) \geq 0, X(0) \geq 0, A(0) \geq 0, R(0) \geq$ 0 , and $M(0) \geq 0$ are positively invariant for system (2).

Proof. Adding the first four equations of (2), we have

$$
\frac{d N}{d t}=\mu-\mu N
$$

It follows that

$$
N(t)=1+N(0) e^{-\mu t}
$$

where $N(0)$ is the initial total number of people. Thus,

$$
\lim _{t \rightarrow \infty} N(t)=1 \text {. }
$$

Then $S(t)+X(t)+A(t)+R(t) \leq 1$; the subpopulation is represented in proportion to the total number of population.

Thus, since the system (2) monitors human population, it is plausible to assume that all its state variables and parameters are nonnegative for all $t \geq 0$. Further, from the last equation of system (2), we have

$$
\begin{aligned}
\frac{d M}{d t} & =m A-\gamma M \\
& \leq m-\gamma M .
\end{aligned}
$$

It follows that

$$
0 \leq M(t) \leq \frac{m}{\gamma}+M(0) e^{-\gamma t},
$$

where $M(0)$ represents the initial value of cumulative density of awareness programs. Thus,

$$
\lim _{t \rightarrow \infty} \sup M(t) \leq \frac{m}{\gamma} .
$$

It implies that the region

$$
\begin{aligned}
& \Omega=\{(S(t), X(t), A(t), R(t), M(t)) \\
& \left.\quad \in R_{+}^{5}: S(t)+X(t)+A(t)+R(t) \leq 1 ; M(t) \leq \frac{m}{\gamma}\right\}
\end{aligned}
$$

is a positively invariant set for (2). So we consider dynamics of system (2) on the set $\Omega$ in this paper.

2.2.2. Positivity of Solutions. For system (2), to ensure that the solutions of the system with positive initial conditions remain positive for all $t>0$, it is necessary to prove that all the state variables are nonnegative, so we have the following lemma.

Lemma 2. If $S(0)>0, X(0)>0, A(0)>0, R(0)>0$, and $M(0)>0$, then the solutions $S(t), X(t), A(t), R(t)$, and $M(t)$ of system (2) are positive for all $t \geq 0$.

Proof. Under the given initial conditions, it is easy to prove that the solutions of the system (2) are positive; if not, we assume a contradiction that there exists a first time $t_{1}$ such that

$$
\begin{gathered}
S(0)>0, \quad S\left(t_{1}\right)=0, \quad S^{\prime}\left(t_{1}\right) \leq 0, \\
X(t)>0, \quad A(t)>0, \quad R(t)>0, \\
M(t)>0, \quad 0 \leq t<t_{1} .
\end{gathered}
$$

In that case, from the first equation of system (2), we have

$$
S^{\prime}\left(t_{1}\right)=\mu+\sigma X>0 .
$$

which is a contradiction meaning that $S(t)>0, t>0$.

Or there exists a $t_{2}$ such that

$$
\begin{gathered}
X(0)>0, \quad X\left(t_{2}\right)=0, \quad X^{\prime}\left(t_{2}\right) \leq 0, \\
S(t)>0, \quad A(t)>0, \quad R(t)>0, \\
M(t)>0, \quad 0 \leq t<t_{2} .
\end{gathered}
$$

In that case, from the second equation of system (2), we have

$$
X^{\prime}\left(t_{2}\right)=\lambda S M>0,
$$

which is a contradiction meaning that $X(t)>0, t \geq 0$.

Or there exists a $t_{3}$ such that

$$
\begin{gathered}
A(0)>0, \quad A\left(t_{3}\right) \leq 0, \quad A^{\prime}\left(t_{3}\right)=0, \\
S(t)>0, \quad X(t)>0, \quad R(t)>0, \\
M(t)>0, \quad 0 \leq t<t_{3} .
\end{gathered}
$$

In that case, from the third equation of system (2), we have

$$
A^{\prime}\left(t_{3}\right)=q R>0 .
$$

which is a contradiction meaning that $A(t)>0, t \geq 0$. Similarly, it can be shown that $R(t)>0$ and $M(t)>0$ for all $t \geq 0$. Thus, the solutions $S(t), X(t), A(t), R(t)$, and $M(t)$ of system (2) remain positive for all $t>0$.

\section{Analysis of the Model}

There are one alcohol free equilibrium $E_{0}$ and one alcohol present equilibrium $E^{*}$ for system (2).

3.1. Alcohol Free Equilibrium and the Basic Reproduction Number. The model has an alcohol free equilibrium given by

$$
E_{0}=(1,0,0,0,0) \text {. }
$$


In the following, the basic reproduction number of system (2) will be obtained by the next generation matrix method formulated in [25].

Let $x=(A, R, M, S, X)^{T}$; then system (2) can be written as

$$
\frac{d x}{d t}=\mathscr{F}(x)-\mathscr{V}(x)
$$

where

$$
\mathscr{V}(x)=\left(\begin{array}{c}
\beta S A \\
0 \\
0 \\
0 \\
0
\end{array}\right),
$$

The Jacobian matrices of $\mathscr{F}(x)$ and $\mathscr{V}(x)$ at the alcohol free equilibrium $E_{0}$ are, respectively,

$$
\begin{gathered}
D \mathscr{F}\left(E_{0}\right)=\left(\begin{array}{cc}
F_{2 \times 2} & 0 \\
0 & 0
\end{array}\right), \\
D \mathscr{V}\left(E_{0}\right)=\left(\begin{array}{ccccc}
V_{2 \times 2} & 0 & 0 & 0 \\
-m & 0 & \gamma & 0 & 0 \\
\beta & 0 & \lambda & \mu & -\sigma \\
0 & 0 & -\lambda & 0 & \sigma+\mu
\end{array}\right),
\end{gathered}
$$

where

$$
F=\left(\begin{array}{cc}
\beta & 0 \\
0 & 0
\end{array}\right), \quad V=\left(\begin{array}{cc}
p+\mu & -q \\
-p & q+\mu
\end{array}\right)
$$

The model reproduction number denoted by $R_{0}$ is thus given by

$$
R_{0}=\frac{\beta(q+\mu)}{(p+\mu)(q+\mu)-p q} .
$$

\subsection{Global Stability of $E_{0}$}

Theorem 3. For system (2), the alcohol free equilibrium $E_{0}$ is globally asymptotically stable if $R_{0} \leq 1$.

Proof. We introduce the following Lyapunov function [26]:

$$
\begin{gathered}
V(S(t), X(t), A(t), R(t), M(t)) \\
\quad=S-\ln S+X+A+\frac{q}{q+\mu} R .
\end{gathered}
$$

The derivative of $V$ is given by

$$
\begin{aligned}
\dot{V}= & \dot{S}-\frac{\dot{S}}{S}+\dot{A}+\frac{q}{q+\mu} \dot{R}+\dot{X} \\
= & \mu-\beta S A-\lambda S M+\sigma X-\mu S \\
& -\frac{1}{S}(\mu-\beta S A-\lambda S M+\sigma X-\mu S) \\
& +\lambda S M-\sigma X-\mu X+\beta S A+q R-p A \\
& -\mu A+\frac{q}{q+\mu}(p A-q R-\mu R) \\
= & \mu-\mu S-\frac{1}{S} \mu+\beta A+\lambda M-\frac{1}{S} \sigma X+\mu \\
& -\mu X+q R-p A-\mu A+\frac{q}{q+\mu} p A-q R \\
= & \mu\left(2-S-\frac{1}{S}\right)+\beta\left(1-\frac{1}{R_{0}}\right) A+\left(1-\frac{1}{S}\right) \sigma X .
\end{aligned}
$$

If $R_{0} \leq 1$, then $\beta\left(1-\left(1 / R_{0}\right)\right) \leq 0$. As we know, $2-S-(1 / S) \leq 0$ and $1-(1 / S) \sigma X \leq 0$, so we obtain $\dot{V} \leq 0$. Furthermore, $\dot{V}=0$ only if $S=1$ or $R_{0}=1$. The maximum invariant set in $\{(S, X, A, R, M): \dot{V}=0\}$ is the singleton $E_{0}$. By Lasalle's invariance principle [27], $E_{0}$ is globally asymptotically stable in $\Omega$.

\subsection{Alcohol Present Equilibrium}

\subsubsection{Existence of the Alcohol Present Equilibrium}

Theorem 4. If $R_{0}>1$, system (2) has a unique alcohol present equilibrium $E^{*}\left(S^{*}, X^{*}, A^{*}, R^{*}, M^{*}\right)$, where

$$
\begin{aligned}
S^{*} & =\frac{1}{\beta}\left(p+\mu-\frac{p q}{q+\mu}\right), \\
X^{*} & =\frac{\lambda m}{\beta \gamma(\sigma+\mu)}\left(p+\mu-\frac{p q}{q+\mu}\right) A^{*}, \\
R^{*} & =\frac{p}{q+\mu} A^{*}, \\
M^{*} & =\frac{m}{\gamma} A^{*} .
\end{aligned}
$$

Proof. It follows from system (2) that

$$
\begin{gathered}
\mu-\beta S A-\lambda S M+\sigma X-\mu S=0, \\
\lambda S M-\sigma X-\mu X=0, \\
\beta S A+q R-p A-\mu A=0, \\
p A-q R-\mu R=0, \\
m A-\gamma M=0 .
\end{gathered}
$$


From the fourth and fifth equations of (27), we obtain

$$
\begin{aligned}
R & =\frac{p}{q+\mu} A, \\
M & =\frac{m}{\gamma} A .
\end{aligned}
$$

Substituting $R$ into the third equation of (27), we have

$$
S=\frac{1}{\beta}\left(p+\mu-\frac{p q}{q+\mu}\right) .
$$

Then substituting (29) and (30) into the second equation of (27), we get

$$
X=\frac{\lambda m}{\beta \gamma(\sigma+\mu)}\left(p+\mu-\frac{p q}{q+\mu}\right) A .
$$

For $A \neq 0$, substituting (29), (30), and (31) into the first equation of (27) gives

$$
\begin{aligned}
\mu- & \mu S \\
= & \left(p+\mu-\frac{p q}{q+\mu}\right) A+\frac{\lambda m}{\beta \gamma}\left(p+\mu-\frac{p q}{q+\mu}\right) A \\
& -\frac{\sigma \lambda m}{\beta \gamma(\sigma+\mu)}\left(p+\mu-\frac{p q}{q+\mu}\right) A .
\end{aligned}
$$

Substituting (30) into (32), we get

$$
\begin{aligned}
A= & \left(\mu-\frac{\mu}{\beta}\left(p+\mu-\frac{p q}{q+\mu}\right)\right) \\
& \times\left(\left(p+\mu-\frac{p q}{q+\mu}\right)\left[1+\frac{\lambda m}{\beta \gamma}-\frac{\sigma \lambda m}{\beta \gamma(\sigma+\mu)}\right]\right)^{-1} \\
= & \mu\left(1-\frac{1}{R_{0}}\right) \\
& \times\left(\left(p+\mu-\frac{p q}{q+\mu}\right)\left[1+\frac{\lambda m}{\beta \gamma}-\frac{\sigma \lambda m}{\beta \gamma(\sigma+\mu)}\right]\right)^{-1} .
\end{aligned}
$$

Therefore, there exists a unique positive root in the interval $(0,1)$ when $R_{0}>1$; there is no positive root in the interval $[0,1]$ when $R_{0} \leq 1$.

For $R_{0}>1$, we carry out the simulation (see Figure 4 ) and give the following conjecture.

Conjecture. If $R_{0}>1$, the alcohol present equilibrium $E^{*}$ of (2) is globally asymptotically stable.

Remark. Since the global stability of (2) is a hard problem, we only carry out simulation (see Figure 4). How to prove the global stability of the alcohol present equilibrium $E^{*}$ of (2) and give the conditions based on model parameters is still an open problem.

\section{Numerical Simulation}

To illustrate the analytic results obtained above, we give some simulations using the parameter values in Table 1. Numerical results are displayed in the following figures. It is reported that binge drinking occurs in up to 30 percent of adolescents and alcohol use disorders occur in about 6 percent of this age group; approximately 50 to 60 percent of them remain abstinent at the end of a year's treatment and a majority of those stay dry permanently $[29,30]$. So the initial condition is taken as the lowest by $A(0)=0.3$ and $R(0)=0.15$; in addition, we assume that $X(0)=0.1, S(0)=0.45$, and $M(0)=0.002$.

First, we choose $P=0.3$ and the numerical simulation gives $R_{0}=0.5691<1$; then the alcohol free equilibrium $E_{0}$ is globally asymptotically stable (Figure 2 ). Second, we choose $P=0.21$ and the numerical simulation gives $R_{0}=1$; the alcohol free equilibrium $E_{0}$ is globally asymptotically stable (Figure 3). Third, we choose $P=0.032$ and the numerical simulation gives $R_{0}=1.165>1$; the alcohol present equilibrium $E^{*}$ is globally asymptotically stable (Figure 4).

Finally, for showing that awareness programs by media can help to control the population of binge drinking, we compare our model with the model which has been studied by Mulone and Straughan [10]; the model in [10] is governed by the following system of nonlinear ordinary differential equations:

$$
\begin{aligned}
& \frac{d S(t)}{d t}=\mu N-\frac{\beta A S}{N}-\mu S, \\
& \frac{d A(t)}{d t}=\frac{\beta A S}{N}+\rho R-(\phi+\mu) A, \\
& \frac{d R(t)}{d t}=\phi A-(\rho+\mu) R .
\end{aligned}
$$

We choose $P=0.032$ and the numerical simulation gives $R_{0}=1.1645>1$; the alcohol present equilibrium $P^{*}$ of (34) is globally asymptotically stable. The number of the binge drinking population is generally on the rise without effects of awareness programs by media.

For showing the distinction between (2) and (34), we take $P=0.032$ and compare the variations of the binge drinking population $A(t)$. We can see that, because of awareness programs by media (Figure 5), the number of people who have alcohol problems decreases due to awareness-induced isolation of susceptible people, so making more people avoid contacting with the heavy drinkers due to the fact that the awareness programs by media can reduce the alcohol problems.

Further, the variations of the binge drinking population $A(t)$ with respect to time $t$ for different values of growth rate of density of awareness programs $m$ are shown in Figure 6. From these figures, it is apparent that as the rate of density of awareness programs $m$ increases the binge drinking population $A(t)$ decreases. Then, the awareness programs by media are an effective measure in alcohol problems. 
TABLE 1: Description and estimation of parameters.

\begin{tabular}{|c|c|c|c|}
\hline$\mu$ & Recruitment rate of the population & 0.25 year $^{-1}$ & {$[10]$} \\
\hline$\beta$ & Transmission coefficient & 0.3 year $^{-1}$ & {$[10]$} \\
\hline$\lambda$ & Dissemination rate of aware individuals & 0.02 year $^{-1}$ & estimate \\
\hline$\sigma$ & Rate of transfer of aware individuals to susceptible class & 0.001 year $^{-1}$ & estimate \\
\hline$p$ & The fraction of $A$ go in to treatment & Variable & Variable \\
\hline$q$ & The fraction of $R$ who relapse into $A$ & 0.8 year $^{-1}$ & [10] \\
\hline$m$ & Growth rate of density of awareness programs & 0.05 year $^{-1}$ & {$[28]$} \\
\hline$\gamma$ & Depletion rate of awareness programs & 0.05 year $^{-1}$ & estimate \\
\hline
\end{tabular}

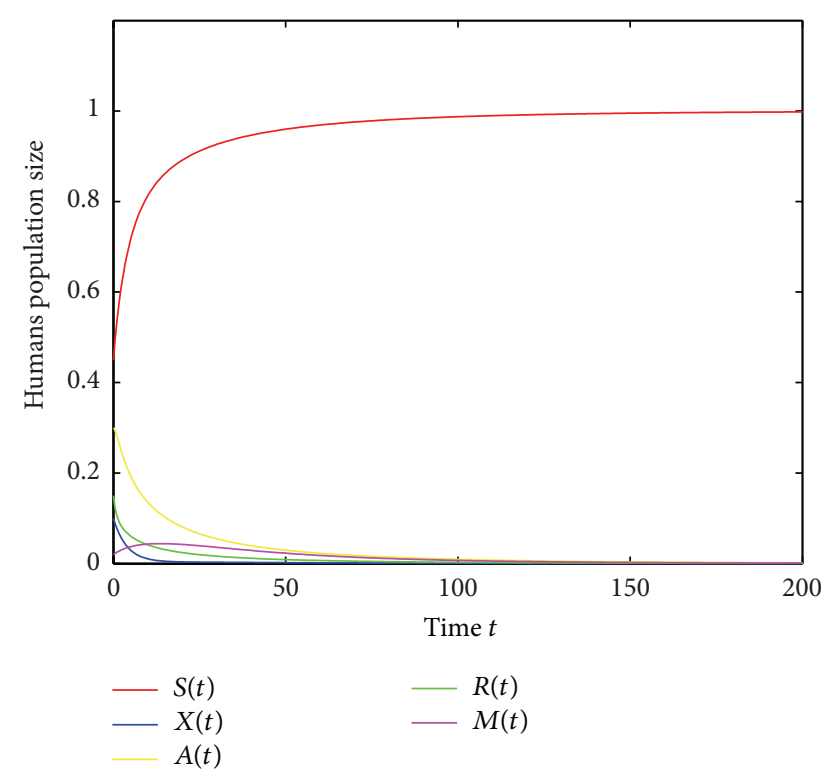

FIGURE 2: If $R_{0}<1$, the alcohol free equilibrium $E_{0}$ is globally asymptotically stable.

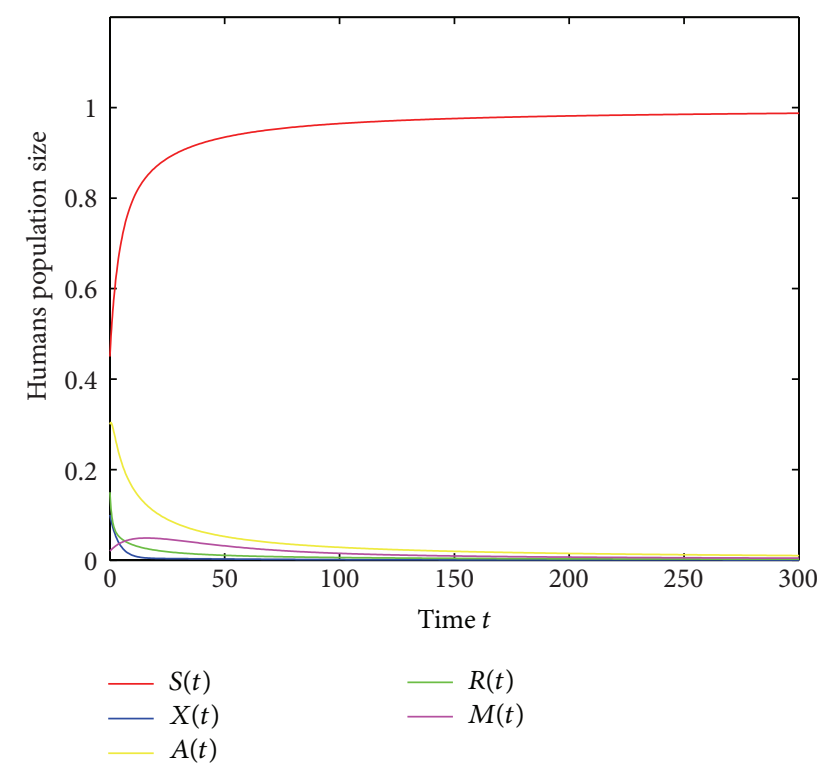

FIgURE 3: If $R_{0}=1$, the alcohol free equilibrium $E_{0}$ is globally asymptotically stable.

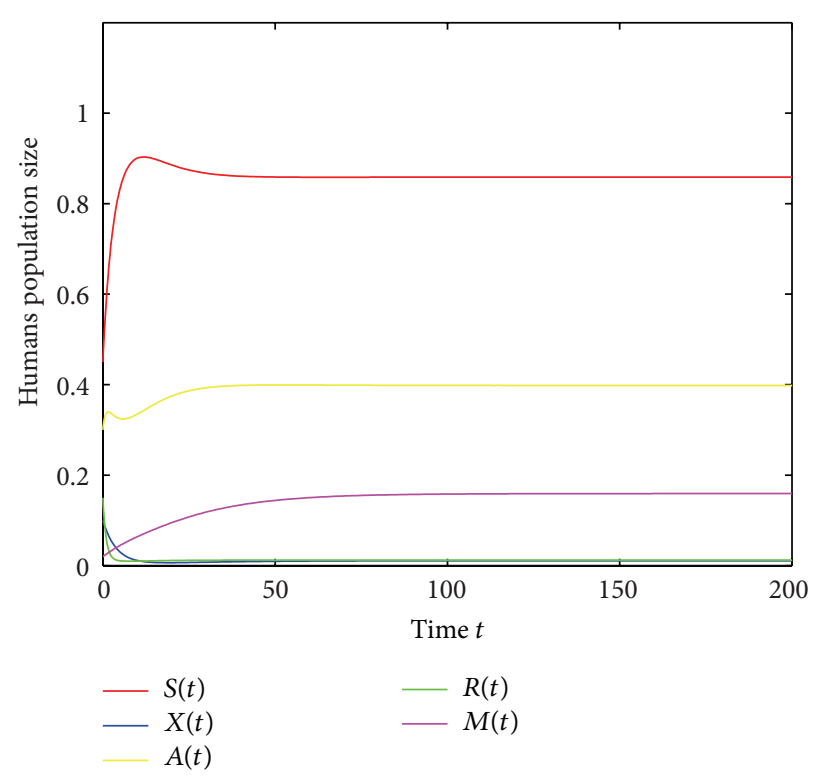

FIGURE 4: If $R_{0}>1$, the alcohol present equilibrium $E^{*}$ is globally asymptotically stable.

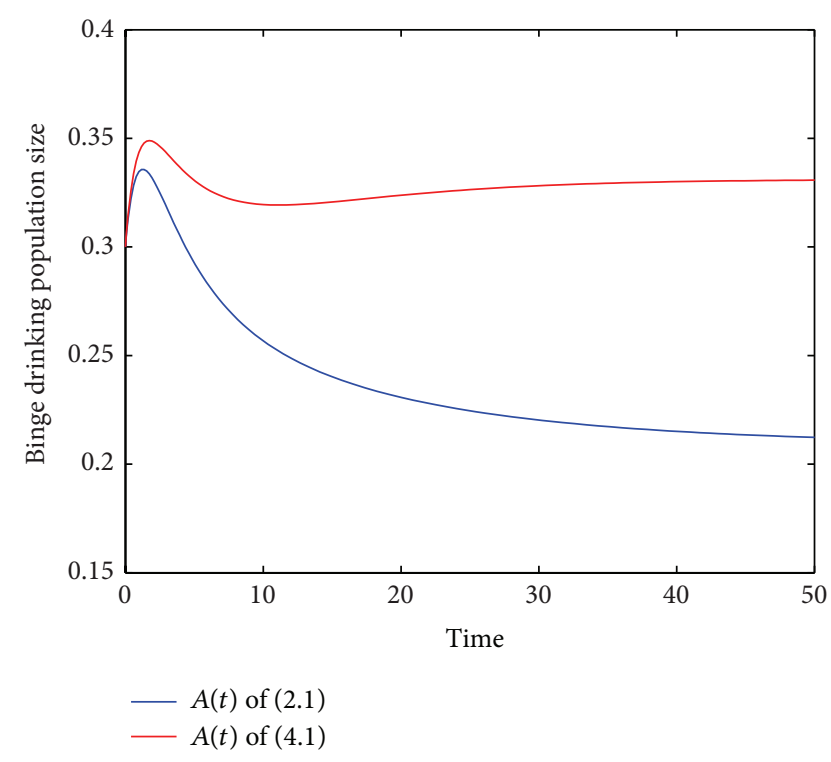

FIGURE 5: Variations of the binge drinking population $A(t)$ of (2) and (34). 


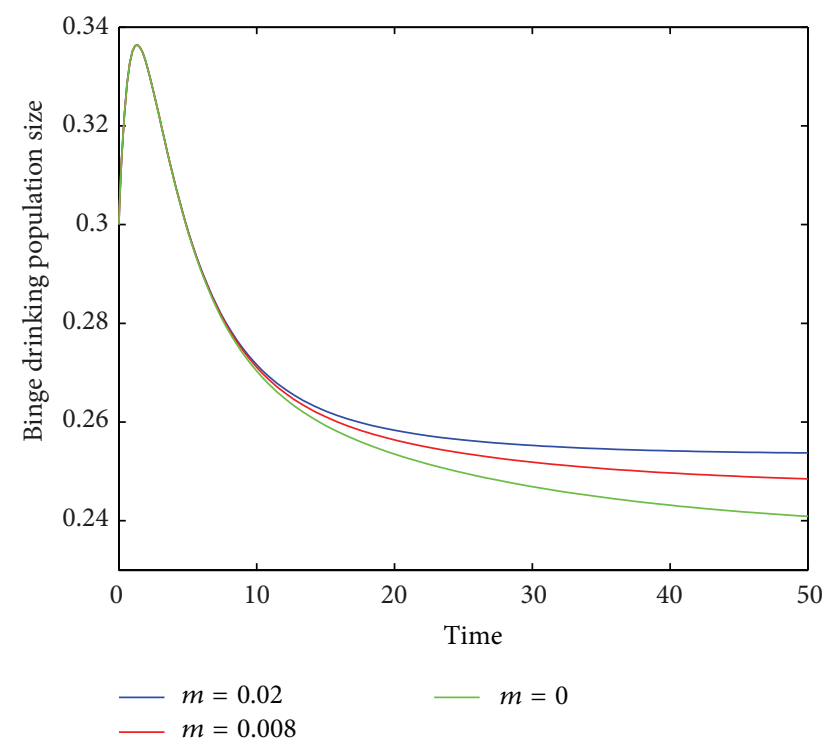

FIGURE 6: Variations of binge drinking population with time for different values of $m$.

\section{Discussion}

We have formulated the effect of awareness programs on the binge drinking and analyzed their dynamical behaviors. The model exhibits two equilibria, explicitly the alcohol free equilibrium and alcohol present equilibrium. By constructing Lyapunov function, we establish some sufficient conditions for the global stability of the alcohol free and the alcohol present equilibria and give some numerical simulations to explain our main result. In our model, we have assumed that the cumulative density of awareness programs by media is proportional to the population of binge drinking heavily. It may be noted that the number of alcohol consumption cases known to the policy makers is sometimes old and thus the number of awareness depends on data. So it is more plausible to consider a time delay in the growth rate of awareness programs [21]. We can modify (2) to the following model with delay:

$$
\begin{aligned}
& \frac{d S(t)}{d t}=\mu-\beta S A-\lambda S M+\sigma X-\mu S, \\
& \frac{d X(t)}{d t}=\lambda S M-\sigma X-\mu X, \\
& \frac{d A(t)}{d t}=\beta S A+q R-p A-\mu A, \\
& \frac{d R(t)}{d t}=p A-q R-\mu R, \\
& \frac{d M(t)}{d t}=m A(t-\tau)-\gamma M .
\end{aligned}
$$

We leave these works for the future.

\section{Conflict of Interests}

The authors declare that there is no conflict of interests regarding the publication of this paper.

\section{Acknowledgments}

This work was partially supported by the NNSF of China (10961018), the NSF of Gansu Province of China (1107RJZA088), the NSF for Distinguished Young Scholars of Gansu Province of China (1111RJDA003), the Special Fund for the Basic Requirements in the Research of University of Gansu Province of China, and the Development Program for Hong Liu Distinguished Young Scholars in Lanzhou University of Technology.

\section{References}

[1] J. Rehm, "The risks associated with alcohol use and alcoholism," Alcohol Research \& Health, vol. 34, no. 2, pp. 135-143, 2011.

[2] J. Rehm, C. Mathers, S. Popova, M. Thavorncharoensap, Y. Teerawattananon, and J. Patra, "Global burden of disease and injury and economic cost attributable to alcohol use and alcohol-use disorders," The Lancet, vol. 373, no. 9682, pp. 22232233, 2009.

[3] J. Ribes, R. Clèries, L. Esteban, V. Moreno, and F. X. Bosch, "The influence of alcohol consumption and hepatitis B and C infections on the risk of liver cancer in Europe," Journal of Hepatology, vol. 49, no. 2, pp. 233-242, 2008.

[4] H. Wechsler and T. F. Nelson, "Focusing attention on college student alcohol consumption and the environmental conditions that promote it," Journal of Studies on Alcohol and Drugs, vol. 69, no. 4, pp. 481-490, 2008.

[5] L. M. Powell, J. Williams, and H. Wechsler, "Study habits and the level of alcohol use among college students," Education Economics, vol. 12, no. 2, pp. 135-149, 2004.

[6] H. Wechsler, B. Moeykens, A. Davenport, S. Castillo, and J. Hansen, "The adverse impact of heavy episodic drinkers on other college students," Journal of Studies on Alcohol and Drugs, vol. 56, no. 6, pp. 628-634, 1995.

[7] S. Mushayabasa and C. P. Bhunu, "Modelling the effects of heavy alcohol consumption on the transmission dynamics of gonorrhea," Nonlinear Dynamics, vol. 66, no. 4, pp. 695-706, 2011.

[8] P. Ormerod and G. Wiltshire, "Binge" drinking in the UK: a social network phenomenon," Mind \& Society, vol. 8, no. 2, pp. 135-152, 2009.

[9] F. Sanchez, X. H. Wang, C. Castillo-Chavez, D. M. Gorman, and P. J. Gruenewald, "Drinking as an epidemica simple mathematical model with recovery and relapse," in Therapists Guide to Evidence-Based Relapse Prevention: Practical Resources for the Mental Health Professional, K. A. Witkiewitz and G. A. Marlatt, Eds., pp. 353-368, Academic Press, Burlington, Canada, 2007.

[10] G. Mulone and B. Straughan, "Modeling binge drinking," International Journal of Biomathematics, vol. 5, no. 1, Article ID 1250005, 14 pages, 2012.

[11] H.-F. Huo and N.-N. Song, "Global stability for a binge drinking model with two stages," Discrete Dynamics in Nature and Society, vol. 2012, Article ID 829386, 15 pages, 2012.

[12] H. F. Huo and C. C. Zhu, "Modeling the effect of constant immigration on drinking behaviour," Submitted. 
[13] H. F. Huo and C. C. Zhu, "Stability of a quit drinking model with relapse," Submitted.

[14] H.-F. Huo and C.-C. Zhu, "Influence of relapse in a giving up smoking model," Abstract and Applied Analysis, vol. 2013, Article ID 525461, 12 pages, 2013.

[15] G. Schwitzer, G. Mudur, D. Henry et al., "What are the roles and responsibilities of the media in disseminating health information?" PLoS Medicine, vol. 2, no. 7, article e215, 2005.

[16] E. Jaramillo, "The impact of media-based health education on tuberculosis diagnosis in Cali, Colombia," Health Policy and Planning, vol. 16, no. 1, pp. 68-73, 2001.

[17] R. Liu, J. Wu, and H. Zhu, "Media/psychological impact on multiple outbreaks of emerging infectious diseases," Computational and Mathematical Methods in Medicine, vol. 8, no. 3, pp. 153164, 2007.

[18] T. P. Palfai, R. Zisserson, and R. Saitz, "Using personalized feedback to reduce alcohol use among hazardous drinking college students: the moderating effect of alcohol-related negative consequences," Addictive Behaviors, vol. 36 , no. 5, pp. 539-542, 2011.

[19] J. M. Tchuenche and C. T. Bauch, "Dynamics of an infectious disease where media coverage influences transmission," ISRN Biomathematics, vol. 2012, Article ID 581274, 10 pages, 2012.

[20] H. Xiang, N. N. Song, and H. F. Huo, "Modelling effects of public health educational campaigns on drinking dynamics," Submitted.

[21] A. K. Misra, A. Sharma, and V. Singh, "Effect of awareness programs in controlling the prevalence of an epidemic with time delay," Journal of Biological Systems, vol. 19, no. 2, pp. 389402, 2011.

[22] A. K. Misra, A. Sharma, and J. B. Shukla, "Modeling and analysis of effects of awareness programs by media on the spread of infectious diseases," Mathematical and Computer Modelling, vol. 53, no. 5-6, pp. 1221-1228, 2011.

[23] "WHO Report of Alcohol in developing societies summary," http://www.who.int/.

[24] D. Chisholm, J. Rehm, M. van Ommeren, and M. Monteiro, "Reducing the global burden of hazardous alcohol use: a comparative cost-effectiveness analysis," Journal of Studies on Alcohol, vol. 65, no. 6, pp. 782-793, 2004.

[25] P. van den Driessche and J. Watmough, "Reproduction numbers and sub-threshold endemic equilibria for compartmental models of disease transmission," Mathematical Biosciences, vol. 180, pp. 29-48, 2002.

[26] J.-Q. Li, Y.-L. Yang, and Y.-C. Zhou, "Global stability of an epidemic model with latent stage and vaccination," Nonlinear Analysis: Real World Applications, vol. 12, no. 4, pp. 2163-2173, 2011.

[27] J. P. LaSalle, "Stability theory for ordinary differential equations," Journal of Differential Equations, vol. 4, no. 1, pp. 57-65, 1968.

[28] L. B. Snyder, "Health communication campaigns and their impact on behavior," Journal of Nutrition Education and Behavior, vol. 39, no. 2, pp. S32-S40, 2007.

[29] D. B. Clark, O. Bukstein, and J. Cornelius, "Alcohol use disorders in adolescents: epidemiology, diagnosis, psychosocial interventions, and pharmacological treatment," Pediatric Drugs, vol. 4, no. 8, pp. 493-502, 2002.

[30] "WebMD Report of Alcohol Abuse Health Center," http://www. webmd.com. 


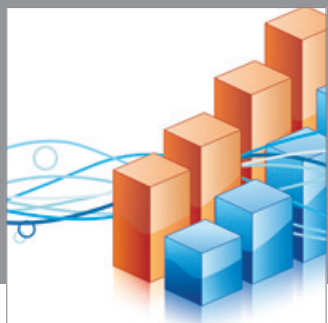

Advances in

Operations Research

mansans

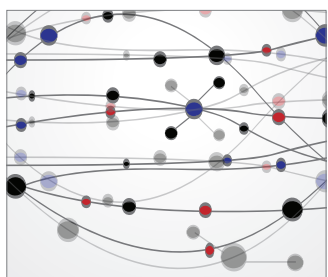

The Scientific World Journal
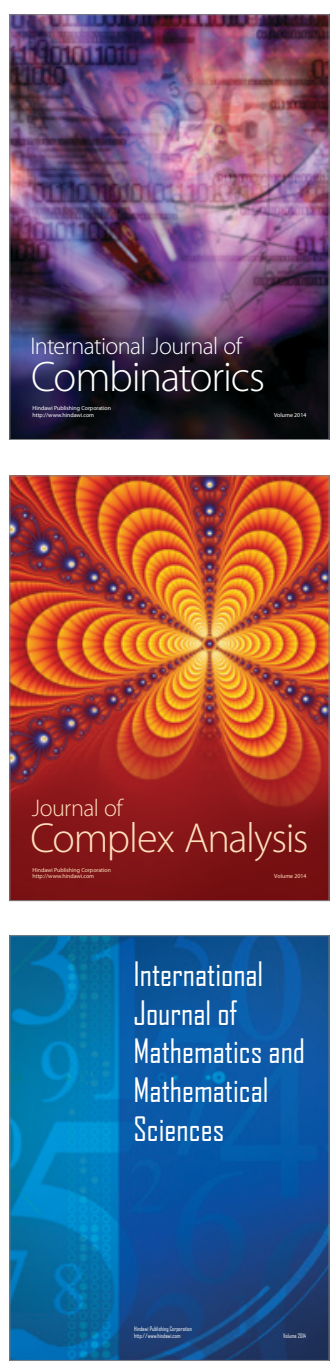
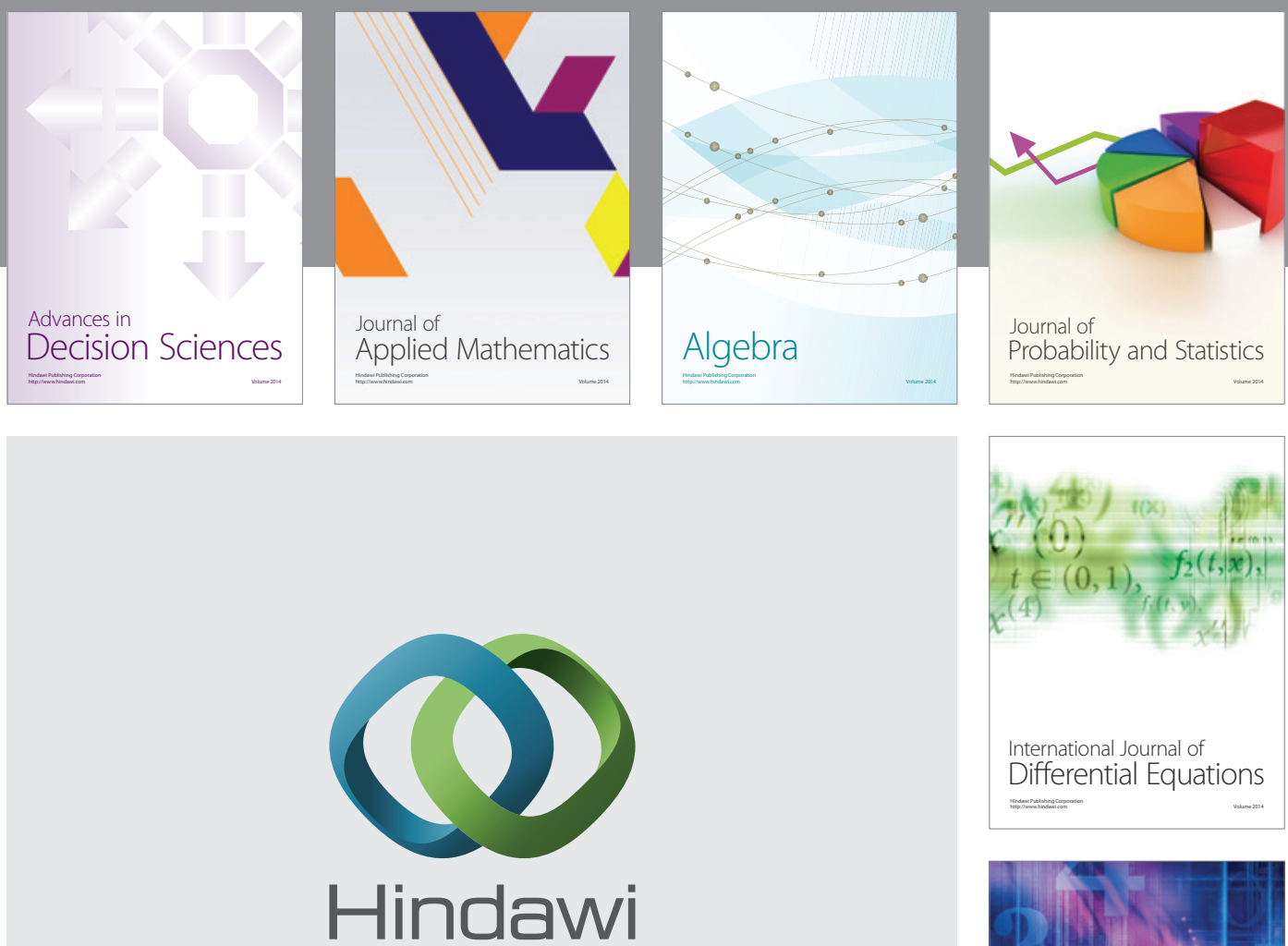

Submit your manuscripts at http://www.hindawi.com
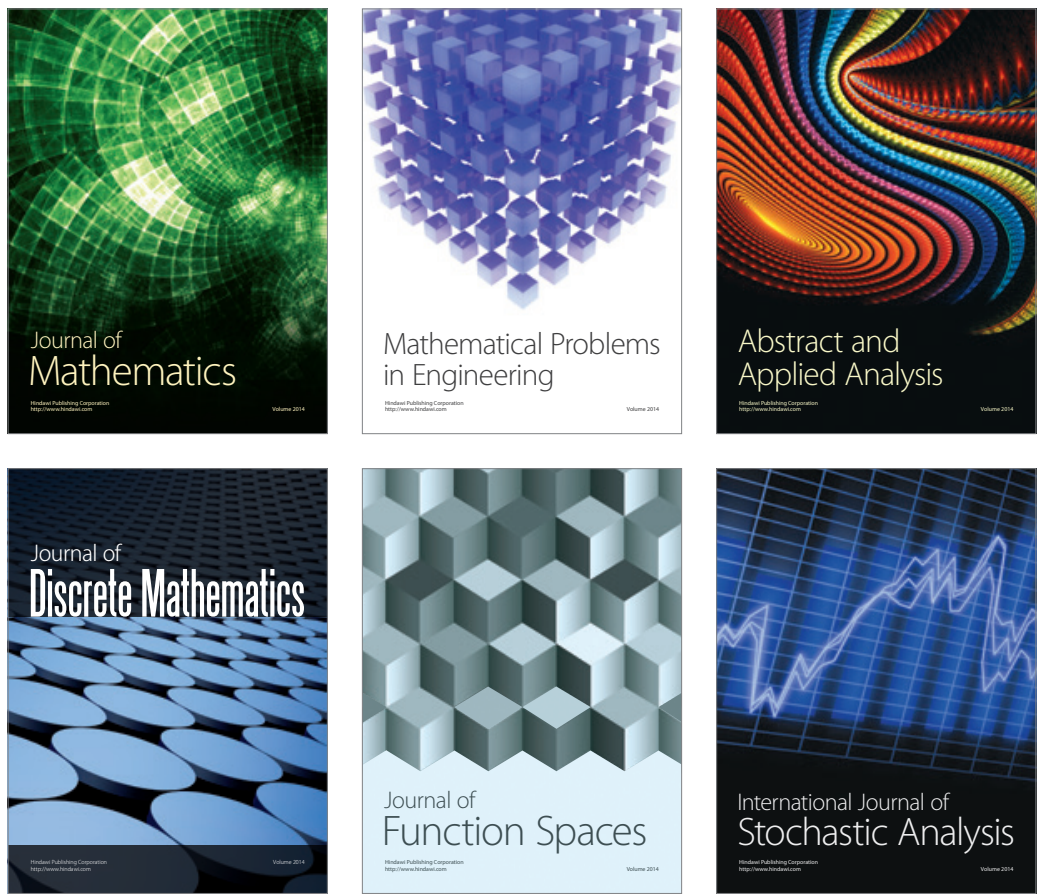

Journal of

Function Spaces

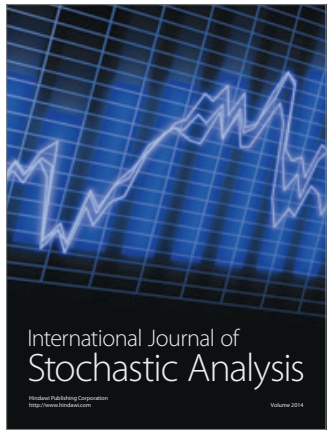

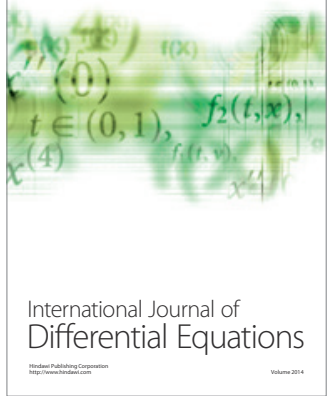
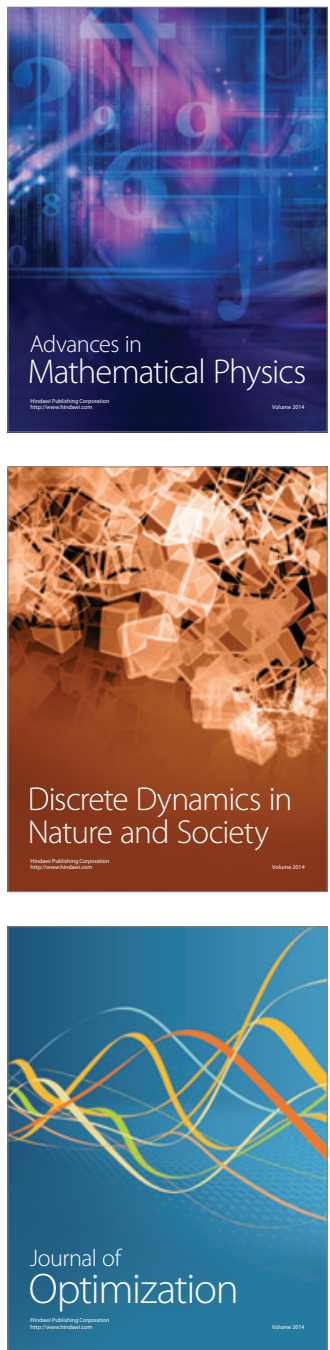